\title{
Alterations in neurotransmitters targeted metabolomics from the key nuclei of brain reward circuits in cocaine-induced behavioral sensitization for self-administering rats
}

\author{
Yang Li \\ Tang Du Hospital \\ Xin Wang \\ Tang Du Hospital \\ Shun-nan Ge \\ Tang Du Hospital \\ Xue-lian Wang ( $\nabla$ tdwxlian@126.com ) \\ Tang Du Hospital
}

\section{Research Article}

Keywords: Targeted Metabolomics, Neurotransmitters, Reward circuits, Behavioral sensitization, Cocaine.

Posted Date: March 19th, 2021

DOl: https://doi.org/10.21203/rs.3.rs-318553/v1

License: (c) (i) This work is licensed under a Creative Commons Attribution 4.0 International License.

Read Full License 


\section{Abstract}

Drug addiction can be considered as a metabolic disease because it is triggered by the disruption of metabolism and causes persistent neurochemical disorders that lead to addiction. The purpose of this study was to identify the potential brain biomarkers and the molecular mechanisms in cocaine-induced behavioral sensitization for self-administering rats. UHPLC-MS/MS targeted metabolomics was used to measure 23 neurotransmitters metabolites which may serve as the biological indices underlying the mechanisms of cocaine-induced behavioral sensitization for the key nuclei of brain reward circuits in the process of cocaine addiction. The measurement results showed that there were many changes for many neurotransmitters metabolites in the key nuclei of brain reward circuits (the striatum, the NAc, the hippocampus, the PFC, etc.), but there was no change in the cerebellum during the reconsolidation of drug addiction memory. These findings strongly indicated that many neurotransmitter metabolites in above key nuclei of brain reward circuits participated in cocaine-induced behavioral sensitization for selfadministering rats. And it may contribute to a better understanding of metabolic changes in the process of cocaine-induced behavioral sensitization and to be helpful to provide a more integrated view of the molecular underpinnings and to ultimately find biomarkers to assist clinical diagnosis and treatment.

\section{Introduction}

Long-term drug use could change brain function and induce a series of following behavioral changes, mainly including compulsive drug-taking and drug-seeking, which could finally develop to drug addiction [1]. Drug addiction is strongly influenced by both personal genetic constitution and environmental factors [2] and it can be considered as a metabolic disease because it is triggered by the disruption of metabolism and causes persistent neurochemical disorders that lead to addiction [3]. Cocaine addiction is a worldwide and serious public health problem, and it is well known that cocaine produces changes in several monoaminergic neurotransmitter systems and has deleterious effects on several metabolic pathways. More and more evidences showed that cocaine could affect synaptic plasticity in the mesolimbic dopamine system, which was very critical to the brain reward processing [4]. The key nuclei of brain reward circuits mainly included the prefrontal cortex (PFC), the nucleus accumbens (NAc), the ventral tegmental area (VTA), the hippocampus, the amygdala and the striatum et, al. The classic mechanism of cocaine addiction is increased dopaminergic transmission and altered glutamate transmission in the striatum $[5,6]$.

In recent years, metabolomics approaches were applied to various fields such as drug discovery $[7,8]$, certain biomarkers research [9-12] and metabolic pathways confirmation [11, 13], due to the ability to detect subtle changes in a large dataset with comprehensive metabolite measurements. Metabolomics is a relatively new and rapidly evolving technology that can capture the effect of an external stimulus on the endogenous metabolome. Metabolomics (also known as metabolic profiling) is the study of metabolism and metabolites in living organisms, and the qualitative and quantitative analysis of those small molecules $(<1,500 \mathrm{Da})$ that are able to change in response to a certain stimulus [14] and which are involved in different metabolic pathways required for the maintenance, growth, and normal function of a 
cell. Metabolomics could link the interactions between genes and the related environment, and it could represent not only the downstream output of the genome, but also the upstream input to the related environment [14-16].

The targeted metabolomics represents the most direct way to gain insights into the metabolomic changes in certain tissue $[17,18]$. Olesti et al. mainly focused on neurotransmitters to successfully predict the pharmacological profile of NPS by this way $[19,20]$. Neurotransmitters are endogenous signaling molecules that play key roles in neuronal communications in transmitting signals from neurons to targeted cells through chemical synapses. Their main function is to convey information throughout the brain and other parts of the body [20]. The neurotransmitters mainly include single amine (e.g. serotonin, dopamine, norepinephrine, epinephrine) and amino acids (e.g. aspartic acid, glutamic acid, glycine, gamma-aminobutyric acid), purine (e.g. adenosine, ATP), choline (e.g. acetylcholine), peptide (e.g. neuropeptide Y) and a certain gas (e.g. NO) [20]. The role of neurotransmitter systems in cocaine addiction, although recognized [21-23], has not been comprehensively evaluated with respect to mechanisms of addiction [24].

The purpose of this study was to identify the potential brain biomarkers and the molecular neurotoxicity mechanisms in cocaine-induced behavioral sensitization for self-administering rats. UHPLC-MS/MS targeted metabolomics was used to measure 23 neurotransmitters metabolites which may serve as the biological indices underlying the mechanisms of cocaine-induced behavioral sensitization for the key nuclei of brain reward circuits in the process of cocaine addiction. These findings may contribute to a better understanding of metabolic changes in the process of cocaine-induced behavioral sensitization and provided a summary of cocaine-induced metabolites, which may be helpful to provide a more integrated view of the molecular underpinnings and to ultimately find biomarkers to assist clinical diagnosis and treatment.

\section{Materials And Methods}

\section{Animals}

All animal procedures in our experiments were consistent with the guidelines of the Committee for Animal Care and Use (No. TDLL2020-03-176, Tangdu Hospital, the Fourth Military Medical University, Xi'an, Shaanxi, China). And the experimental protocols were approved by the Committee for Animal Care and Use of Tangdu Hospital, the Fourth Military Medical University. Male Sprague-Dawley rats (initial weight: $300-350 \mathrm{~g})$ were individually housed in an animal center at a controlled temperature $\left(21 \pm 2^{\circ} \mathrm{C}\right)$ and humidity (40-60\%) with a 12-hour light-dark cycle (lights on at 07:00) and were provided with water ad libitum.

\section{Drug preparation}

Cocaine hydrochloride (Qinghai Pharmaceutical Co. Ltd., Xining, Qinghai, China), which was dissolved in saline to a final concentration of $8 \mathrm{mg} / \mathrm{mL}$ and was stored at room temperature away from light.

\section{Jugular vein catheterization}


The rats were anesthetized with sodium pentobarbital (50 mg/ $\mathrm{kg}$ body weight, i.p.) and a sterile special silicone tube was guided into the right jugular vein for every rat. Following surgery, all rats were immediately housed individually in feeding cages, and allowed to recover for at least 3 days. And the special silicone tube was rinsed daily with saline containing heparin and antibiotics.

\section{Drug self-administration apparatus}

The size of drug self-administration (SA) apparatus (AES-101, AniLab Software \& Instruments Co., Ltd., Ningbo, Zhejiang, China) is $40 \mathrm{~cm} \times 40 \mathrm{~cm} \times 50 \mathrm{~cm}$. The bottom of this apparatus is a bottom plate (like a fence) with a strip hole. Two nose-pokes (left nose-poke and right nose-poke) are on the wall about 5 $\mathrm{cm}$ away from the bottom of the apparatus. The system can receive an input signal from the nose-poke when the experimental rat's nose is probed into a nose-poke hole, and then it can produce many output signals as our requirements. Three signal lights (green light, red light, and white light) and a buzzer are individually upon the apparatus wall. The top of the apparatus is a lever with a delivery catheter and a shaft connection. To protect it, a spring tube is out of the delivery catheter. The upper end of the catheter is connected to the injection pump through the liquid phase shaft, and the lower end will be connected to the rat's jugular vein catheterization. In addition, there is a camera on the top of the box to record the conditions during the training of rats.

According to the experimental requirement, we need to edit the appropriate experimental method (Fixed ratio, $F R=1)$. When the rat performs a valid nose-poke, the system will pump drug once ( $T=1.25 \mathrm{~s}, v=$ $1.60 \mathrm{~mL} / \mathrm{min}$ ), accompanied with several changes in environmental cues such as signal lights (Green light is off, and red light is on for $20 \mathrm{~s}$ ), valid nose-poke light ( $T=20 \mathrm{~s}$ ), and sound (buzzer, $T=1.25 \mathrm{~s}$ ). Once a valid nose-poke is completed, the system will immediately enter a 20-s refractory period. During this period, the rat's nose-poke behavior (called invalid nose-poke) will also be recorded, but no systemic output reaction will be triggered. After the refractory period expires, the system will complete a cycle, and then it will enter the next cycle (Signal lights: Red light is off, green light is on).

\section{Drug self-administration training procedure}

After 3 days recovery following the jugular vein catheterization surgery, animals would go to the selfadministration training procedure, which consisted of a 14-days training schedule. When the experimental rat finished a valid nose-poke, it could receive a small food prize while being administrated drug (cocaine) via its right jugular vein, companied with several changes in environmental cues such as signal lights (Green light is off, and red light is on for $20 \mathrm{~s}$ ), valid nose-poke light ( $T=20 \mathrm{~s}$ ), and sound (buzzer, $T=1.25$ s). The rats were trained for 2 hours per day, and the number of different nose-pokes and the number of pumps was all recorded.

\section{Tissue preparation}

After the last 2-hours cocaine self-administration training, the experimental rats were deeply anesthetized with sodium pentobarbital ( $50 \mathrm{mg} / \mathrm{kg}$ body weight, i.p.). The caudate putamen/striatum (CPu), the nucleus accumbens (NAc), the prefrontal cortex (PFC), the hippocampus and the cerebellum were 
immediately dissected according to the brain atlas (Paxinos and Franklin, 2004) and frozen in liquid nitrogen before storage at $-80^{\circ} \mathrm{C}$ until next processing.

The brain tissue samples were homogenized with $1 \mathrm{~mL}$ tissue grinder dounce (Wheaton, USA). The brain tissue samples were processed in $400 \mu \mathrm{L} 80 \%$ acetonitrile aqueous solution (formic acid at $0.1 \%,-20{ }^{\circ} \mathrm{C}$ ). After adding steel ball, $35 \mathrm{~Hz}$ grinding for 4 minutes, and ultrasonic for 5 minutes (ice water). Then repeat the grinding and ultrasonic step 3 times, and precipitate at $-20^{\circ} \mathrm{C}$ overnight. The mixture was centrifuged (15 min, $12,000 \mathrm{rpm}, 4^{\circ} \mathrm{C}$ ) and $80 \mu \mathrm{L}$ supernatant was put into $40 \mu \mathrm{L} 100 \mathrm{mmol} / \mathrm{L}$ sodium carbonate solution and $40 \mu \mathrm{L} 2 \%$ benzoyl chloride solution, swirl and mix for 30 min at room temperature, then add $40 \mu \mathrm{L} 1 \%$ formic acid solution to adjust $\mathrm{pH}$ to weak acid. Finally, the mixture was centrifuged (10 min, $12,000 \mathrm{rpm}, 4^{\circ} \mathrm{C}$ ) and the supernatant was put into LC injection flask and kept at $-20^{\circ} \mathrm{C}$ until UHPLCMS/MS analysis.

\section{Statistical analysis}

All results were expressed as the means \pm SEM. The data were statistically assessed via the Student's two-tailed t-test and one-way ANOVA test using SPSS 18.0 (SPSS Inc., USA). $P$-values are marked statistically significant as follows: ${ }^{\star} P<0.05, \star \star P<0.01, \star \star \star * P<0.001$.

\section{Results}

\section{Effective establishment of cocaine self-administration (SA) model for freely moving rats}

The classical self-administration (SA) model for freely moving rats is one of many effective and important methods for studying drug addiction. In our study, when the number of valid nose-poke increased significantly and reached a relatively stable level in the end of training period (within varying less than $15 \%$ for three consecutive days), the drug SA model for freely moving rats would be basically considered to be successful.

During cocaine SA training, many rats might be removed because several complications (e.g. leaking, plugging, serious infection, etc.). In total, we had a statistical analysis on the rats' behavior according to the number of valid nose-poke for the first three days $\left(\operatorname{Day}_{1-3}\right)$ and the last three days (Day $\left.{ }_{12-14}\right)$ in the Saline group $(n=8)$ and Cocaine group $(n=9)$, respectively. There was a significant trend of decreasing for that in the Saline group ( $t_{\text {Saline group }}=3.528, P<0.001$; Fig. $1 \mathrm{~A}$ and $\mathrm{B}$ ), and the number of valid nosepoke reached a relatively stable level during the last three days of training period $\left(\mathrm{F}_{\text {Saline group }}=2.891, P=\right.$ 0.0778; Fig. 1C). Compared to the first three days, we found that the number of valid nose-poke for the last three days in the Cocaine group increased significantly $\left(\mathrm{t}_{\text {Cocaine group }}=11.37, P<0.001\right.$; Fig. $1 \mathrm{D}$ and $\mathrm{E}$ ) during cocaine SA training period and reached a relatively stable level during the last three days of training period $\left(\mathrm{F}_{\text {Cocaine group }}=0.1555, P=0.8569 ; \mathrm{Fig} .1 \mathrm{~F}\right)$. In addition, compared with the Saline group, the number of valid nose-poke for the last three days in the Cocaine group significantly increased $\left(\mathrm{t}_{\text {Cocaine }}\right.$ group, Saline group $=27.47, P<0.001 ;$ Fig. $1 \mathrm{G}$ ), which indicating that the Cocaine group had effectively established cocaine self-administration model. Figure $1 \mathrm{H}$ showed the original recordings of the 
experimental rat's behavioral events. The drug self-administration training lasted 2 hours per day, and the number of nose-pokes (left nose-poke and right nose-poke) and the number of pumps were all recorded. Figure 1/ showed a schematic diagram about cocaine self-administration training for freely moving rats.

\section{The changes of targeted metabolomics in cocaine addiction}

The UHPLC-MS/MS targeted metabolomics method was used to analyze and detect the brain tissue samples from the key nuclei in reward circuits of 85 drug-addicted rats, aiming at measuring 23 neurotransmitters metabolites in the samples. Compared to the Saline group, the statistical results of 19 neurotransmitters metabolites from the key nuclei of reward circuits in the Cocaine group were as follows (Fig. 2 and Table 1):

1) The striatum: L-Histidine increased significantly, while 5-Hydroxyindoleacetic acid, L-Kynurenine, Homovanillic acid, L-Dopa and 3,4-Dihydroxyphenylacetic Acid all decreased.

2) The NAc: Acetylcholine, L-Histidine, L-Arginine, Histamine, L-Glutamine increased significantly, while Homovanillic acid decreased.

3) The PFC: L-Histidine and L-Glutamic acid increased significantly.

4) The hippocampus: L-Arginine and L-Glutamine increased significantly.

5) The cerebellum: there was no obvious changes.

The results showed that there were many changes for many neurotransmitters metabolites in the key nuclei of brain reward circuits (the striatum, the NAc, the hippocampus, the PFC), but there was no change in the cerebellum. From the results of neurotransmitter targeted metabolomics, many neurotransmitters metabolites in the key nuclei of brain reward circuits (the striatum, the NAc, the hippocampus, the PFC) participated in the process of cocaine-induced behavioral sensitization for self-administering rats.

Above figures showed 19 neurotransmitters metabolites from the key nuclei of reward circuits (A-S) in the process of cocaine-induced behavioral sensitization for self-administering rats. Compared to the Saline group, 19 neurotransmitters metabolites from the key nuclei of reward circuits in the Cocaine group. SA: Self-administration. Data were presented as means \pm SEM, ${ }^{* * \star} P<0.001$. 
Table 1

Statistical results of 23 neurotransmitters metabolites from the key nuclei in reward circuits

\begin{tabular}{|c|c|c|c|c|c|c|}
\hline No. & Compound Name & $\mathrm{CPu}$ & NAC & PFC & Hippocampus & Cerebellum \\
\hline 1 & Acetylcholine & प & $\uparrow^{*}$ & प & 0 & प \\
\hline 2 & L-Histidine & $\uparrow^{*}$ & $\uparrow^{*}$ & $\uparrow^{*}$ & Q & 口 \\
\hline 3 & L-Arginine & प & $\uparrow * *$ & 口 & $\uparrow^{*}$ & 口 \\
\hline 4 & Histamine & प & $\uparrow * \star \star$ & 口 & ૫ & ૫ \\
\hline 5 & L-Glutamine & प & $\uparrow^{*}$ & प & $\uparrow^{*}$ & Q \\
\hline 6 & L-Glutamic acid & प & प & $\uparrow^{*}$ & प & ૫ \\
\hline 7 & 4-Aminobutyric acid & $\square$ & Q & प & Q & ૫ \\
\hline 8 & Vanillylmandelic acid & $\square$ & $\square$ & प & Q & 0 \\
\hline 9 & Melatonin & N.D. & N.D. & N.D. & N.D. & N.D. \\
\hline 10 & L-Tryptophan & प & प & प & प & प \\
\hline 11 & 5-Hydroxyindoleacetic acid & $\downarrow^{\star *}$ & प & प & प & प \\
\hline 12 & Tryptamine & N.D. & N.D. & N.D. & N.D. & N.D. \\
\hline 13 & L-Kynurenine & $\downarrow^{*}$ & प & प & प & प \\
\hline 14 & L-5-Hydroxytryptophan & प & प & प & प & प \\
\hline 15 & Homovanillic acid & $\downarrow^{*}$ & $\downarrow^{*}$ & प & प & प \\
\hline 16 & L-Tyrosine & प & 0 & प & 0 & 0 \\
\hline 17 & L-Dopa & $\downarrow^{*}$ & 0 & प & 0 & 0 \\
\hline 18 & 3,4-Dihydroxyphenylacetic Acid & $\downarrow^{*}$ & प & प & प & प \\
\hline 19 & 5-Hydroxytryptamine & $\square$ & प & प & 口 & 口 \\
\hline 20 & Norepinephrine & प & 口 & 口 & प & प \\
\hline 21 & Tyramine & N.D. & N.D. & N.D. & N.D. & N.D. \\
\hline 22 & Adrenaline & N.D. & N.D. & N.D. & N.D. & N.D. \\
\hline 23 & Dopamine & प & प & प & 0 & प \\
\hline
\end{tabular}




\section{Discussion}

In this experiment, by detecting the changes in behavioral biology and targeted metabolomics in the brain reward circuits, we demonstrated the functional activation of the brain reward circuits via a classical cocaine-induced self-administration (SA) model for freely moving rats. In our experiment, UHPLC-MS/MS targeted metabolomics was used to determine 23 neurotransmitters metabolites. The measurement results showed that there were many changes for many neurotransmitters metabolites in the key nuclei of brain reward circuits (the striatum, the NAc, the hippocampus, the PFC, etc.), but there was no change in the cerebellum during the reconsolidation of drug addiction memory. These findings strongly indicated that many neurotransmitter metabolites in the key nuclei of brain reward circuits (the striatum, the NAc, the hippocampus, the PFC) participated in the process of cocaine-induced behavioral sensitization for self-administering rats.

Addiction can be considered a metabolic disease because it is triggered by the destruction of metabolism and causes persistent neurochemical disorders, which leads to addiction [3]. Another previous study showed that after intraperitoneal injection of cocaine, serotonin, norepinephrine, glucose, dopamine, 3, 4dihydroxy Phenylacetic acid (DOPAC) and 5-hydroxyindoleacetic acid in several brain regions such as thalamus, striatum and frontal cortex, have undergone significant changes [25]. In addition, the study also showed that cocaine has an effect on thalamic glycolysis [25].

The classic mechanism of cocaine addiction is increased dopaminergic transmission and altered glutamate transmission in the striatum $[5,6]$. Previous drug abuse studies using metabolomics methods have demonstrated the potential of metabolomics and the concept of a multi-event mechanism for cocaine addiction. Patkar et al. used a liquid chromatography electrochemical array platform to perform metabolomics analysis on cocaine-dependent plasma samples and drug-free plasma samples, and found that $\mathrm{N}$-methyl serotonin and guanine were significantly up-regulated, while hypoxanthine and Xanthine is down-regulated $[26,27]$. Li et al. used $1 \mathrm{H}-\mathrm{NMR}$ to study metabolomics in cocaine rat brain tissue $[27,28]$, and the result showed that changes in tissue concentrations of creatine, taurine, and Nacetylaspartate were observed after cocaine treatment, meanwhile providing metabolic changes related to neurotransmitters, oxidative stress, and mitochondrial disorders.

Exploring changes in neurotransmitters in the key nuclei of brain reward circuits in the process of cocaine addiction by targeted metabolomics, our experimental results were as follows:

1) Striatum: L-Histidine increases, while 5-Hydroxyindoleacetic acid, L-Kynurenine, Homovanillic acid, LDopa and 3,4-Dihydroxyphenylacetic Acid all decreased.

2) Nucleus accumbens: Acetylcholine, L-Histidine, L-Arginine, Histamine, L-Glutamine increased significantly, while Homovanillic acid decreased.

3) Prefrontal cortex (PFC): L-Histidine and L-Glutamic acid increased significantly.

4) Hippocampus: L-Arginine and L-Glutamine increased significantly. 
5) Cerebellum: there was no obvious changes.

The results showed that there were many changes for many neurotransmitters metabolites in the key nuclei of brain reward circuits (the striatum, the NAc, the hippocampus, the PFC, etc.), but there was no change in cerebellum. From the results of neurotransmitter targeted metabolomics, many neurotransmitter metabolites in the key nuclei of brain reward circuits (the striatum, the NAc, the hippocampus, the PFC) participated in the process of cocaine addiction.

Although the role of the neurotransmitters system in cocaine addiction has been recognized [21-23], the mechanism of toxicity and addiction has not been fully evaluated $[24,26]$. Similar studies have shown that repeated administration of MAP can cause obvious neurotransmitters disturbances, oxidative stress, and membrane destruction in the hippocampus, NAc, and PFC. These metabolites can be used as biological indicators of the MAP-induced behavior sensitization mechanism. Environmental factors could affect specific proteins and metabolites, and these chemicals could become potential biomarkers, help diagnose addiction, and could even be used as treatment targets.

Although a large number of proteins and metabolites changes related to drug abuse have been detected, so far, it has been difficult to identify a certain molecule as a potential biomarker. There was increasing evidence that these metabolites were inherently involved in multiple metabolic pathways in our body, and the relative quantification of metabolites in biological fluids could provide the organism's overall metabolic status and provide new insights into the underlying mechanisms of disease. Using metabolomics to identify disturbances in biochemical processes may provide a deeper understanding of the biological and molecular pathways of exposure, addiction, and withdrawal, meanwhile helping to reveal potential biomarkers for diagnosis or monitoring treatment.

According to the neurotransmitters metabolomics results, many neurotransmitter metabolites in the key nuclei of brain reward circuits (the striatum, the NAc, the hippocampus, the PFC, etc.) participated in the process of cocaine addiction. Our experiment only showed the phenomenon of neurotransmitters metabolites changes, and no further and deeper research has been done on the neurotransmitter metabolic pathways, nor do we know how to explain the mechanism in depth, but it aimed to provide a new perspective for the study of drug addiction. Above findings may contribute to a better understanding of metabolic changes in the process of cocaine-induced behavioral sensitization and provided a summary of cocaine-induced metabolites, which may be helpful to provide a more integrated view of the molecular underpinnings and to ultimately find biomarkers to assist clinical diagnosis and treatment.

\section{Declarations}

\section{Acknowledgements}

This project was supported by the National Natural Science Foundation of China (No. 81971244 and 81671366, awarded to Xue-lian Wang) and Science and Technology Innovation Foundation of Tangdu Hospital, the Fourth Military Medical University (No. 2017LCYJ002, awarded to Xue-lian Wang). 


\section{Conflicts of interest}

The authors have no conflicts of interest to declare.

\section{Statement}

All animal procedures in our experiments were consistent with the guidelines of the Committee for Animal Care and Use (No. TDLL2020-03-176, Tangdu Hospital, the Fourth Military Medical University, Xi'an, Shaanxi, China). And the experimental protocols were approved by the Committee for Animal Care and Use of Tangdu Hospital, the Fourth Military Medical University. In addition, all methods in our experiment are reported in accordance with ARRIVE guidelines.

\section{References}

1. Potenza, M.N., et al., Neuroscience of behavioral and pharmacological treatments for addictions. Neuron, 2011. 69(4): p. 695-712.

2. Robison, A.J. and E.J. Nestler, Transcriptional and epigenetic mechanisms of addiction. Nat Rev Neurosci, 2011. 12(11): p. 623-37.

3. Dole, V.P., Narcotic addiction, physical dependence and relapse. N Engl J Med, 1972. 286(18): p. 98892.

4. Ungless, M.A., et al., Single cocaine exposure in vivo induces long-term potentiation in dopamine neurons. Nature, 2001. 411(6837): p. 583-7.

5. Robbins, T.W. and B.J. Everitt, Limbic-striatal memory systems and drug addiction. Neurobiol Learn Mem, 2002. 78(3): p. 625-36.

6. Kalivas, P.W. and C. O'Brien, Drug addiction as a pathology of staged neuroplasticity. Neuropsychopharmacology, 2008. 33(1): p. 166-80.

7. Mercier, K.A., et al., A Metabolomics Pilot Study on Desmoid Tumors and Novel Drug Candidates. Sci Rep, 2018. 8(1): p. 584.

8. Lu, Y. and C. Chen, Metabolomics: Bridging Chemistry and Biology in Drug Discovery and Development. Current Pharmacology Reports, 2017. 3(1): p. 16-25.

9. Klein, M.S. and J. Shearer, Metabolomics and Type 2 Diabetes: Translating Basic Research into Clinical Application. J Diabetes Res, 2016. 2016: p. 3898502.

10. Wang, X., S. Chen, and W. Jia, Metabolomics in Cancer Biomarker Research. Current Pharmacology Reports, 2016. 2(6): p. 293-298.

11. Zhang, A., et al., Mass spectrometry-based metabolomics: applications to biomarker and metabolic pathway research. Biomed Chromatogr, 2016. 30(1): p. 7-12.

12. Ambati, C.S., et al., Identification and Quantitation of Malonic Acid Biomarkers of In-Born Error Metabolism by Targeted Metabolomics. J Am Soc Mass Spectrom, 2017. 28(5): p. 929-938. 
13. Ren, S., et al., Integration of Metabolomics and Transcriptomics Reveals Major Metabolic Pathways and Potential Biomarker Involved in Prostate Cancer. Mol Cell Proteomics, 2016. 15(1): p. 154-63.

14. Steuer, A.E., L. Brockbals, and T. Kraemer, Metabolomic Strategies in Biomarker Research-New Approach for Indirect Identification of Drug Consumption and Sample Manipulation in Clinical and Forensic Toxicology? Front Chem, 2019. 7: p. 319.

15. Wishart, D.S., Emerging applications of metabolomics in drug discovery and precision medicine. Nat Rev Drug Discov, 2016. 15(7): p. 473-84.

16. Zaitsu, K., et al., Application of metabolomics to toxicology of drugs of abuse: A mini review of metabolomics approach to acute and chronic toxicity studies. Drug Metab Pharmacokinet, 2016. 31(1): p. 21-26.

17. Budczies, J. and C. Denkert, Tissue-Based Metabolomics to Analyze the Breast Cancer Metabolome. Recent Results Cancer Res, 2016. 207: p. 157-75.

18. Gonzalez-Riano, C., A. Garcia, and C. Barbas, Metabolomics studies in brain tissue: A review. J Pharm Biomed Anal, 2016. 130: p. 141-168.

19. Olesti, E., et al., Metabolomics predicts the pharmacological profile of new psychoactive substances. J Psychopharmacol, 2019. 33(3): p. 347-354.

20. Olesti, E., et al., Quantification of endogenous neurotransmitters and related compounds by liquid chromatography coupled to tandem mass spectrometry. Talanta, 2019. 192: p. 93-102.

21. Engblom, D., et al., Glutamate receptors on dopamine neurons control the persistence of cocaine seeking. Neuron, 2008. 59(3): p. 497-508.

22. Oleson, E.B., et al., Dopamine uptake changes associated with cocaine self-administration. Neuropsychopharmacology, 2009. 34(5): p. 1174-84.

23. Patkar, A.A., et al., Relationship of the serotonin transporter with prolactin response to metachlorophenylpiperazine in cocaine dependence. J Psychiatr Res, 2008. 42(14): p. 1213-9.

24. Kalivas, P.W. and N.D. Volkow, The neural basis of addiction: a pathology of motivation and choice. Am J Psychiatry, 2005. 162(8): p. 1403-13.

25. Kaplan, K.A., et al., Neuronal metabolomics by ion mobility mass spectrometry: cocaine effects on glucose and selected biogenic amine metabolites in the frontal cortex, striatum, and thalamus of the rat. Anal Bioanal Chem, 2013. 405(6): p. 1959-68.

26. Patkar, A.A., et al., Alterations in tryptophan and purine metabolism in cocaine addiction: a metabolomic study. Psychopharmacology (Berl), 2009. 206(3): p. 479-89.

27. Zhang, X., et al., Neuronal metabolomics by ion mobility mass spectrometry in cocaine selfadministering rats after early and late withdrawal. Anal Bioanal Chem, 2016. 408(16): p. 4233-45.

28. Li, Y., et al., (1)H NMR-based metabonomics in brain nucleus accumbens and striatum following repeated cocaine treatment in rats. Neuroscience, 2012. 218: p. 196-205.

\section{Figures}



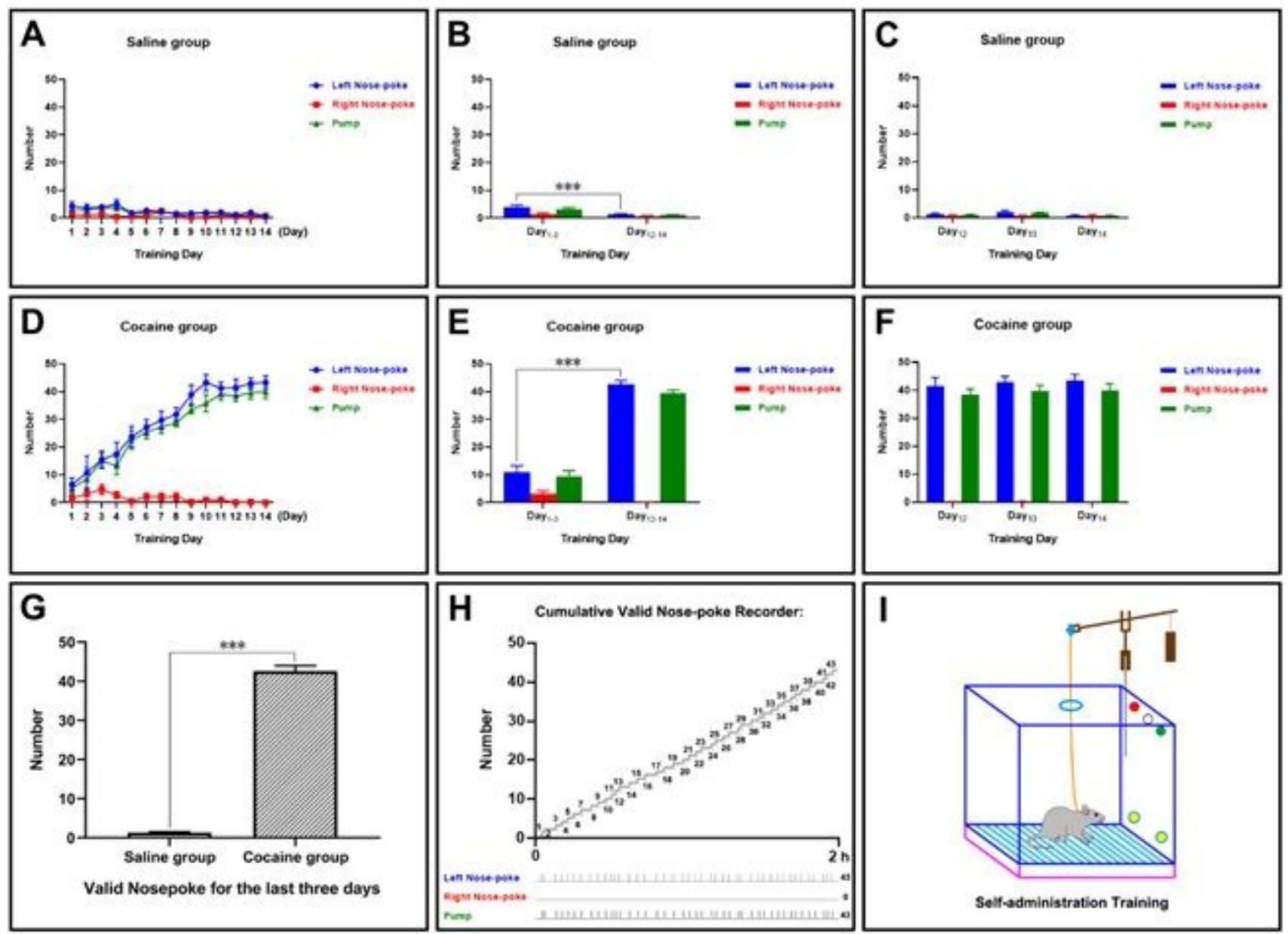

Figure 1

The drug (saline (A) and cocaine (D)) self-administration training procedure. Compared to the first three days, the number of valid nose-poke for the last three days in the Cocaine group increased significantly (Day1-3: $10.93 \pm 4.164$, Day12-14: $42.56 \pm 2.422$, tCocaine group $=11.37, \mathrm{P}<0.001$. (E)) during cocaine SA training period and kept relatively stable during the last three days of training period (FCocaine group = $0.1555, P=0.8569$. (F)). However, there was a little decreasing for that in the Saline group (Day1-3: 4.000 \pm 1.247 , Day12-14: $1.333 \pm 0.3993$, tSaline group $=3.528, P<0.001$. (B)), and the number of valid nosepoke reached a relatively stable level during the last three days of training period (FSaline group $=2.891$, $P=0.0778$. (C)). G. Compared with the Saline group, the number of valid nose-poke for the last three days in the Cocaine group significantly increased (Day12-14 in the Saline group: 1.333 \pm 0.3993 , Day12-14 in the Cocaine group: $42.56 \pm 2.422$, tCocaine group, Saline group $=27.47, \mathrm{P}<0.001)$. H. The original recordings of the experimental rat's behavioral events. The drug self-administration training lasted 2 hours per day, and the number of nose-pokes (left nose-poke and right nose-poke) and the number of pumps were all recorded. I. The cocaine self-administration training for freely moving rats. SA: Selfadministration. Data were presented as means \pm SEM, ${ }^{\star *} \mathrm{P}<0.001$. 


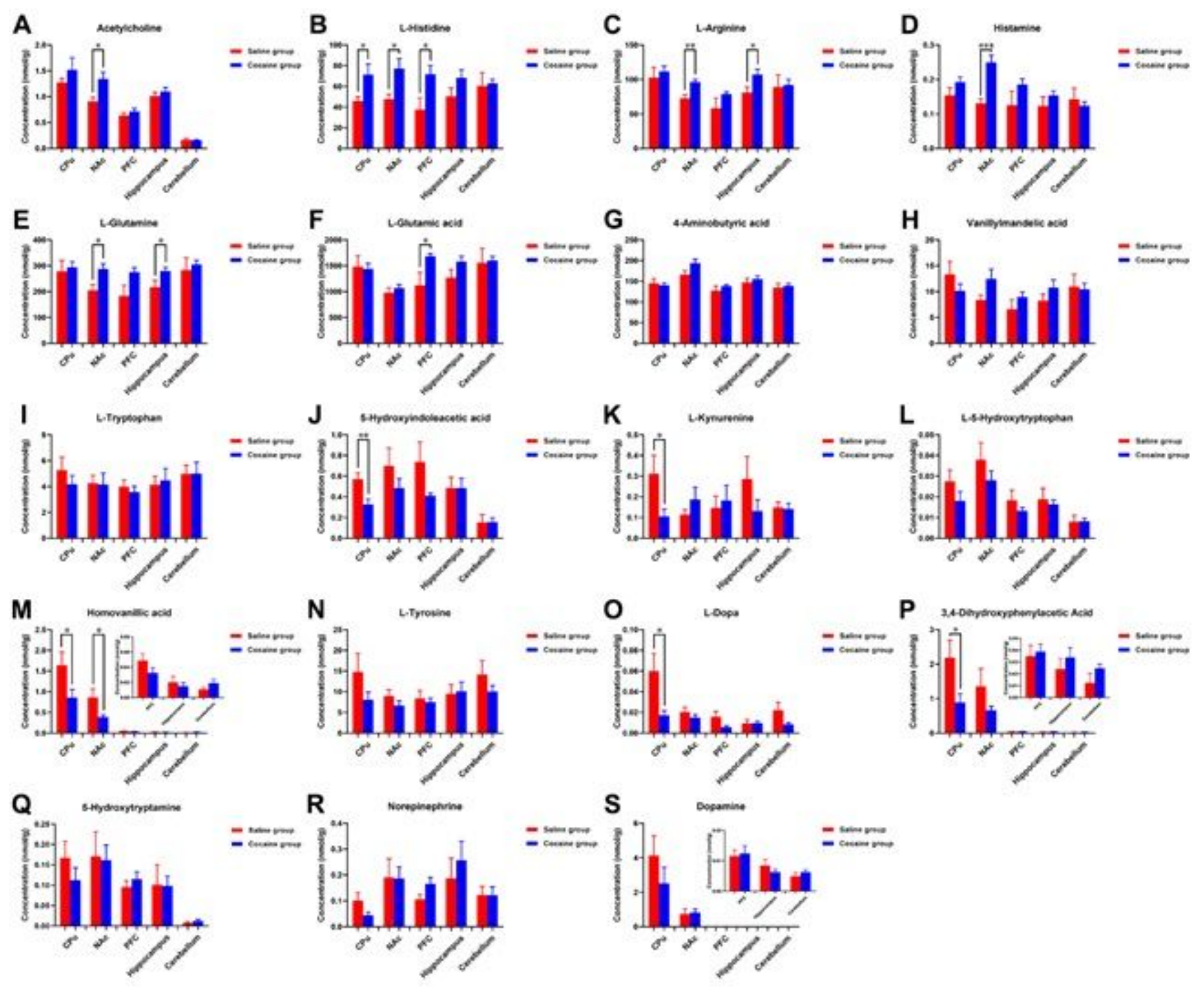

Figure 2

Statistical results of 19 neurotransmitters metabolites from the key nuclei in reward circuits Above figures showed 19 neurotransmitters metabolites from the key nuclei of reward circuits (A-S) in the process of cocaine-induced behavioral sensitization for self-administering rats. Compared to the Saline group, 19 neurotransmitters metabolites from the key nuclei of reward circuits in the Cocaine group. SA: Selfadministration. Data were presented as means \pm SEM, $* \star \star P<0.001$. 